\title{
A Continuum Model for Route Optimization in Large-Scale Inhomogeneous Multi-Hop Wireless Networks
}

\author{
Dene A. Hedges, Justin P. Coon, Senior Member, IEEE, and Gaojie Chen, Senior Member, IEEE
}

\begin{abstract}
Multi-hop route optimization in large-scale inhomogeneous networks is typically NP-hard, for most problem formulations, requiring the application of heuristics which, despite their relatively low processing complexity, find sub-optimal solutions. Where optimal solutions can be determined by Lagrangian based constrained optimization techniques for example, the processing complexity typically scales like $O\left(N^{3}\right), N$ being the number of relays employed. Here, we propose an alternative approach to route optimization by considering the limit of infinite relay node density to develop a continuum model, which yields an optimized equivalent continuous relay path. The model is carefully constructed to maintain a constant connection density even though the node density scales without bound. This leads to a formulation for minimizing the end-to-end outage probability that can be solved using methods from the calculus of variations. With the continuum model, we show that the processing complexity scales linearly with the number of points that sample the continuous path, which can be lower than the number of relay nodes in a large scale network. We demonstrate the effectiveness of this new approach and its potential by considering a network subjected to point sources of interference.
\end{abstract}

Index Terms-Multi-hop relaying, RNPP, calculus of variations, outage, continuum modeling, interference.

\section{INTRODUCTION}

$\mathbf{T}$ HE most prominent evolution of wireless networks is the densification of network deployment [1]-[3]. Further, the unprecedented growth in Internet of Things (IoT) services [4], [5], driven by a number of technological, social, and economic factors [6], will fuel further densification. While the heterogeneous integration of wireless networks is likely to provide the required levels of connection ubiquity between humans, machines, and devices, it will lead to inhomogeneous spatial interference characteristics.

Multi-hop relaying has long been studied as a way to extend coverage in wireless networks, reduce energy consumption, and improve the overall quality of service (QoS). For example, multi-hop network concepts have been applied in IoT networks [7], vehicular networks [8] and numerous publications addressing the challenges associated with Device-to-Device (D2D) communications in future cellular systems (see, e.g., [9] and references therein). Multi-hop connectivity has also been

This work was supported by EPSRC under Grants EP/N002350/1 ("Spatially Embedded Networks") and EP/R006377/1 ("M3NETs") .

D.A. Hedges and J. P. Coon are with the Department of Engineering Science, University of Oxford, Oxford OX1 3PJ, U.K. (e-mail: dene.hedges@eng.ox.ac.uk; justin.coon@eng.ox.ac.uk).

G. Chen is with the Department of Engineering, University of Leicester, Leicester LE1 7RH, U.K. (email: gaojie.chen@leicester.ac.uk). considered in the context of secrecy [10] which investigates the secrecy connectivity probability in multi-hop ad-hoc networks in the presence of inhomogeneous eavesdropper clusters. Also, Chen et al. studied optimal routing based on the trusted connectivity probability for multi-hop D2D communications with decode-and-forward relaying in [11]. But, these latter works only focused on the optimal routing based on the snapshot of the networks, which did not consider how to locate the positions of the relays to achieve the optimal performance when we have a large number of relays.

The majority of previous works that consider multi-hop connectivity are typically in the context of wireless sensor networks (WSN) [12] or cellular networks [13]-[16] where the design goal is to optimize some functional, such as coverage, data throughput or latency for example, and non-functional such as data integrity. These optimization challenges are often further compounded by constraints such as total energy consumption or monetary budget, for example. In the multihop context, these problems are often referred to as the relay node placement problem (RNPP) where the objective relates to the deterministic placement of the minimum number of relay nodes that meet the design objectives. The RNPP has been proven to be NP-hard [17]-[19] and even with a relatively low number of relays, the problem is non trivial and can also be NP-complete [20], [21]. This optimization challenge has been extensively researched and the common theme is the non-trivial nature of the optimization problem that necessitates heuristic or meta-heuristic [22], [23] techniques to deliver a sub-optimal solution. Arriving at an optimal solution, if possible, is often computationally expensive [24]. Survey papers [12], [25], with references therein, provides an overview of heuristic strategies and techniques for node placement in wireless sensor networks. Two key observations can be made from these: the appropriateness and practicality of the heuristic solution, from a numerous set of options, is very much dependent upon the problem scenario and that processing efficiency of the routing algorithm is traded against the optimality of the final solution. If the sub-optimality is acceptable then the low complexity of the heuristic solution is clearly attractive; some heuristic processes can approach a linear scaling [26].

In our work we consider the scenario whereby the placement of $N$-relays is deterministic and where the computation of network performance is polynomial time solvable to yield optimal solutions (in contrast to aforementioned heuristic approaches), for example by Lagrangian formulation of a constrained opti- 
mization problem. Although robust in determining the optimal solution, they are computationally expensive; requiring matrix manipulation that can scale like $O\left(N^{3}\right)$ [27], although this processing complexity can be reduced by an order of magnitude by using augmented Lagrangian methods that do not require matrix manipulations [28]. Although a number of numerical optimization tools exist for this purpose, e.g. the Matlab ${ }^{\circledR}$ function fmincon [29], such tools are computational unwieldy and prohibitive when applied in the context of large (e.g. 100 s or 1000s of relays [1], [30]), energy-constrained relay networks or where networks are subject to temporal variation necessitating continuous re-routing to maintain performance optimality.

In order to yield optimal solutions through lower complexity computation, we consider an alternate method in the form of a continuous equivalent relay path. Our approach is based upon the idea of treating the multi-hop route as a continuum ${ }^{1}$. We argue that this approach has the potential to reduce the complexity in the node placement/route selection process for dense networks. In order to provide a practical context to our work, we consider that the network is subject to spatial inhomogeneity; arising from point sources of co-channel interference $^{2}$. Prior work that has considered continuum modelling include [32] and [33], which treated discrete base station (BS) entities as a continuum of transmitters in order to yield a closed form expression for certain network performance characteristics, and [34], which discussed the application of variational calculus to a problem of determining the minimal exposure path in wireless sensor networks. However, to the best of the authors' knowledge, no prior art exists relating to the use of continuum modelling for route optimization in large scale multi-hop wireless networks. As such, we believe that the proposed model presents a radically different view of wireless networks, which we hope will stimulate further interest in developing new approaches to treating complex wireless networks in the future, even down to the microscopic level, e.g., nanoscale wireless [35] and molecular communications networks [36].

Although exploratory in nature, this research offers the following contributions:

1) we develop a continuum model that describes the endto-end outage probability in a multi-hop relay network;

2) we apply the model to find an analytic solution for the outage-optimal device locations when the system is subject to an inhomogeneous scalar field of interference;

3) the efficacy of the continuum model is verified by considering a discrete sampling of the continuum with a finite number of relay nodes.

The remainder of the paper is organized as follows. In section II, we introduce the discrete optimization based upon the Lagrangian method to provide a baseline for comparison. In section III, we introduce the continuum model for end-toend outage probability calculation. In section IV, we elaborate

\footnotetext{
${ }^{1}$ Continuum mechanics [31] is a field of study whereby accurate behavior models are developed based on the assumption that the substance of an object is assumed to completely fill the space it occupies, even though it actually comprises a large finite number of molecules.

${ }^{2}$ Although other forms of spatial inhomogeneity can be considered.
}

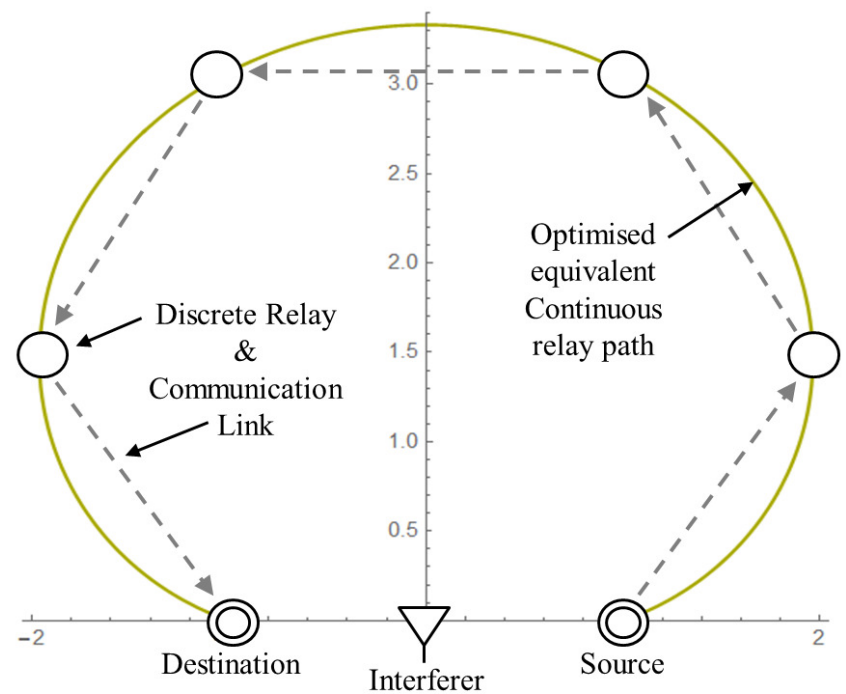

Fig. 1: Multihop wireless network subject to single interferer.

on our continuum model by applying it to study the outageoptimization problem for a multi-hop wireless network subject to a single interferer. This is followed in section $\mathrm{V}$ where we consider multiple interferers along with the effectiveness of approximating a finite number of spatially distributed interferers by a single equivalent interferer. Section VI provides thoughts on how to sample the continuum to obtain discrete node positions for use in practical, finite systems. Conclusions are drawn in section VII.

\section{DisCRETE Optimization OF RELAY LOCATIONS}

Consider the goal of transmitting a message from a source node to a destination node via $N$ relay nodes located in Euclidean space ${ }^{3} \mathbb{R}^{d}, d \in\{2,3\}$. We denote the positions of the source and destination nodes by $\mathbf{p}_{0}$ and $\mathbf{p}_{N+1}$, respectively, and the position of the $i$ th relay node by $\mathbf{p}_{i}{ }^{4}$. For convenience, we collect the positions of the relay nodes in the set $\mathcal{R}=\left\{\mathbf{p}_{1}, \mathbf{p}_{2}, \ldots, \mathbf{p}_{N}\right\}$.

Fig. 1 illustrates the scenario where a communications network, located in $\mathbb{R}^{2}$, is subjected to a scalar field of interference originating from a point source. We assume that all node transmissions are co-channel and temporally continuous ${ }^{5}$, relays employ perfect self interference cancellation [37], [38] and interference from non-adjacent relay nodes is assumed negligible.

In this example, the average interference level at any node will have a spatial dependence on its location. Given known locations of the source, destination and interferer nodes, our goal will be to optimize a performance metric by the appropriate positioning of relay nodes. Here, we chose to minimize the

\footnotetext{
${ }^{3}$ The analysis does not preclude the use of alternative topological spaces. ${ }^{4}$ We use $\mathbf{p}_{i}$ to refer to the $i$ th node, itself, as well as its position in $\mathbb{R}^{d}$.

${ }^{5}$ The subsequent analysis can also be applied to systems employing time division duplex protocols.
} 
outage probability of the source-destination communication link, as defined by

$$
\mathcal{R}^{*}=\underset{\mathcal{R}}{\operatorname{argmin}} \mathrm{f}(\mathcal{R}), \text { s.t. }\left\{\begin{array}{l}
\mathrm{g}(\mathcal{R})=b \\
\mathrm{~h}(\mathcal{R}) \leqslant c
\end{array}\right.
$$

where $\mathcal{R}^{*}$ represents the set of optimized relay node locations, $\mathrm{f}(\mathcal{R})$ is the objective function dependent upon the relay, source, destination and interferer locations, $\mathrm{g}(\mathcal{R})=b$ is an equality constraint function relating to the boundary conditions imposed by fixed positions of the source and destination nodes, $\mathrm{h}(\mathcal{R}) \leqslant c$ represents an arbitrary inequality constraint function and $b$ and $c$ are constants. A more complex scenario may have many more constraints. A Lagrangian formulation is then defined by

$$
\mathcal{L}\left(\mathcal{R}, \lambda_{1}, \lambda_{2}\right)=\mathrm{f}(\mathcal{P})+\lambda_{1}(b-\mathrm{g}(\mathcal{R}))+\lambda_{2}(c-\mathrm{h}(\mathcal{R})),
$$

where $\lambda_{1}$ and $\lambda_{2}$ are Lagrange multipliers. Stationary points are then determined by solving

$$
\nabla \mathcal{L}\left(\mathcal{R}, \lambda_{1}, \lambda_{2}\right)=0
$$

where $\nabla$ is the gradient (vector differential) operator. Solving (3) requires the solution of $N d+2$ simultaneous equations, which is typically done numerically using successive approximation techniques such as Newton's method, which yields the estimate update

$$
\mathrm{x}_{k+1}=\mathrm{x}_{k}-\left[\mathrm{H} \mathcal{L}\left(\mathrm{x}_{k}\right)\right]^{-1} \nabla \mathcal{L}\left(\mathrm{x}_{k}\right), k \geqslant 0,
$$

where $\mathrm{H} \mathcal{L}\left(\mathrm{x}_{\mathbf{k}}\right)$ is the Hessian matrix (second-order partial derivatives of the Lagrangian with respect to the elements of $\mathrm{x}_{k}$ ) and $\mathrm{x}_{k}=\left\{\mathcal{R}_{k}, \lambda_{1_{k}}, \lambda_{2_{k}}\right\}$.

From an initial approximation for the variables, $x_{0}$, we iterate (4) until an acceptable level of convergence is achieved. Since, at every iteration, the inverse of the Hessian matrix needs to be evaluated we have a processing complexity that scales like $\mathcal{O}\left(N^{3}\right)$, assuming a standard algorithm such as Gaussian elimination is employed for matrix inversion [27]. The cubic scaling can be improved by using state-of-the-art algorithms, e.g. Coppersmith-Winograd [39], to approximately $\mathcal{O}\left(N^{2.4}\right)$. Even with these algorithms, however, it is clear that, for large numbers of relays, the discrete optimization of relay locations presents an unwieldy processing task.

\section{CONTINUUm MOdel FOR End-TO-End OUTAGE PROBABILITY}

Given the aforementioned cubic scaling in processing complexity with the number of relay nodes using a discrete model, we postulate that this processing load may be reduced by considering a continuum model whereby we take the limit of infinite relay nodes to derive a continuous relay path. Our goal is to develop a formulation of the end-to-end outage probability for the source-destination link that is a functional of the curve in $\mathbb{R}^{d}$ along which relay devices are placed, i.e., the points in $\mathcal{R}$ should be contained in (or be very close to) the curve. This curve will effectively signify a path of infinite node density. To maintain a practical viewpoint, however, we will retain a finite connection density in the model. It is important to note that without this consideration, the end-to-end outage probability would converge to zero as $N \rightarrow \infty$. To give a better picture of this approach, Fig. 1 illustrates a continuous path along with discrete node placements for a system subject to a point source of interference. We will return to this example later.

\section{A. General Formulation}

To make progress, we consider a wireless network in $\mathbb{R}^{2}$ and a model whereby a connection between two arbitrary points $\mathbf{p}_{i}$ and $\mathbf{p}_{j}$ exists with probability $c\left(\mathbf{p}_{i}, \mathbf{p}_{j}\right)$, which is independent of all other connections. We then form a multi-hop route, between a source $\mathbf{p}_{0}$ and a destination $\mathbf{p}_{N+1}$, via the sequence of relays at the points in $\mathcal{R}$. The probability that an end-to-end connection exists between the source and destination can be written as

$$
P_{c}=\prod_{n=0}^{N} c\left(\mathbf{p}_{n}, \mathbf{p}_{n+1}\right) .
$$

We define the outage probability as the complement of the connection probability, i.e., $P_{o}=1-P_{c}$. It will be convenient to define the logarithmic connection probability (LCP) as

$$
\psi_{c}:=\log P_{c}=\sum_{n=0}^{N} \log c\left(\mathbf{p}_{n}, \mathbf{p}_{n+1}\right) .
$$

We now consider the homogeneous and inhomogeneous cases separately.

\section{B. Homogeneous System}

In the homogeneous case, the connection function between nodes depends only upon the Euclidean distance between them. In this case, we can write

$$
\begin{aligned}
\psi_{c} & =\sum_{n=0}^{N} \log c\left(\left\|\mathbf{p}_{n+1}-\mathbf{p}_{n}\right\|\right) \\
& =\sum_{n=0}^{N} \frac{\log c\left(\left\|\mathbf{p}_{n+1}-\mathbf{p}_{n}\right\|\right)}{\left\|\mathbf{p}_{n+1}-\mathbf{p}_{n}\right\|}\left\|\mathbf{p}_{n+1}-\mathbf{p}_{n}\right\|,
\end{aligned}
$$

where $\|\cdot\|$ is the $l^{2}$-norm. We define $\ell_{n}:=\left\|\mathbf{p}_{n+1}-\mathbf{p}_{n}\right\|$. Any line in $\mathbb{R}^{2}$ can be partitioned into a sequence of points $\mathcal{P}=\left\{\mathbf{p}_{0}, \ldots, \mathbf{p}_{N+1}\right\}$ with $\mathbf{s}=\mathbf{p}_{0}$ denoting the source and $\mathbf{d}=\mathbf{p}_{N+1}$ denoting the destination such that $\ell_{0}+\cdots+\ell_{n} \leq L$, the length of the line. Let the mesh of the partition be defined as $\delta_{\ell}:=\max \ell_{i}$. Suppose as $\delta_{\ell} \rightarrow 0$, we have

$$
\frac{\log c\left(\left\|\mathbf{p}_{n+1}-\mathbf{p}_{n}\right\|\right)}{\left\|\mathbf{p}_{n+1}-\mathbf{p}_{n}\right\|} \rightarrow-q, \quad \forall n \text { and } q \in \mathbb{R}_{+} .
$$

Note that this limit is not dependent upon $N$ since the system is homogeneous. It follows that

$$
\bar{\psi}=\lim _{\delta_{\ell} \rightarrow 0} \psi_{c}=-q \int \mathrm{d} \ell=-q L .
$$

Thus, we can write the outage probability along the line as

$$
p_{o}=1-\mathrm{e}^{-q L} .
$$

It is clear that the path with the minimum length yields the lowest outage probability. This result is consistent with findings in [40] which discusses a relay selection strategy 
where the security of a multi-hop network, in the presence of a homogeneous spatial distribution of eavesdroppers, is optimal when relays are chosen that are closest to the midpoint between source and destination nodes i.e. the resultant multihop path length is minimised.

\section{Inhomogeneous System}

For the case of an inhomogeneous system, we will find it useful to specify point locations in a parametric manner. Define the tagged partition $\mathcal{P}_{z}^{\zeta}=\left\{z_{0}, z_{1}, \ldots, z_{N+1}\right\}$ with $0=z_{0}<z_{1}<\cdots<z_{N+1}=1$ along with a sequence $\zeta_{0}, \ldots, \zeta_{N}$, the elements of which satisfy $\zeta_{n} \in\left[z_{n}, z_{n+1}\right]$. The path of points that the communication will traverse can be written as the sequence $\mathbf{p}(0), \mathbf{p}\left(z_{1}\right), \ldots, \mathbf{p}\left(z_{N+1}\right)$ where $\mathbf{p}(z)=(x(z), y(z))$. We can no longer say that the connection function is only dependent upon the pairwise distance, so we must write

$$
\begin{aligned}
\psi_{c} & =\sum_{n} \log c\left(\mathbf{p}\left(z_{n}\right), \mathbf{p}\left(z_{n+1}\right)\right) \\
& =\sum_{n} \frac{\log c\left(\mathbf{p}\left(z_{n}\right), \mathbf{p}\left(z_{n+1}\right)\right)}{z_{n+1}-z_{n}}\left(z_{n+1}-z_{n}\right) .
\end{aligned}
$$

Let the mesh of the partition $\mathcal{P}_{z}^{\zeta}$ be denoted by $\delta_{z}=$ $\max _{n}\left(z_{n+1}-z_{n}\right)$. Now suppose that as $\delta_{z} \rightarrow 0$, we have

$$
\frac{\log c\left(\mathbf{p}\left(z_{n}\right), \mathbf{p}\left(z_{n+1}\right)\right)}{z_{n+1}-z_{n}} \rightarrow-q\left(\zeta_{n}\right)<0 .
$$

for some function $q:[0,1] \rightarrow \mathbb{R}_{+}$. It follows that if the function $q$ is Riemann integrable, then

$$
\bar{\psi}=\lim _{\delta_{z} \rightarrow 0} \psi_{c}=-\int_{0}^{1} q(z) \mathrm{d} z .
$$

The outage probability in this case can be written as

$$
p_{o}=1-\mathrm{e}^{-\int_{0}^{1} q(z) \mathrm{d} z} .
$$

We note that this result generalizes that presented in (10).

\section{Multi-hop Route Optimization in the Presence OF A SINGLE INTERFERER}

We now proceed to apply our continuum model to optimize the placement of relays, in a multi-hop communications network, when communication takes place in the presence of an inhomogeneous scalar field of interference in $\mathbb{R}^{2}$ caused by a single interferer at a known location (see Fig. 1). Our current analysis focuses on the outage probability of the multi-hop communication link; however, other metrics can be considered.

In order to progress, we must obtain the function $q(z)$. The formulation of $q$ is dependent upon the connection model, which is governed by the channel fading statistics, modulation and coding schemes employed, and various other characteristics of the system including the interferer in this scenario. In what follows, we develop the model further by assuming each pairwise communication link is independent and affected by Rayleigh fading, and the mean path loss follows an inverse power law, with exponent $\eta$, with respect to the Euclidean distance between devices. We firstly consider the connectivity between two arbitrary nodes, transmitter $\mathbf{p}_{i}$ and receiver $\mathbf{p}_{j}$ separated by distance $d_{i j}=\left\|\mathbf{p}_{i}-\mathbf{p}_{j}\right\|$. In this particular example, we can arbitrarily center the coordinate system on the interference source, located at $(0,0)$. The interferer transmits with power $P_{I}$ at a distance $d_{j}=\left\|\mathbf{p}_{j}\right\|$ from the receiving node. We assume that all nodes have antennas with uniform gain $G$ in the $\mathbb{R}^{2}$-plane.

The signal to noise and interference ratio (SINR) at the receiving node will be given by

$$
\mathrm{SINR}_{\mathrm{j}}=\frac{P G^{2}\left(\frac{\lambda}{4 \pi}\right)^{2} d_{i j}^{-\eta}\left|h_{i j}\right|^{2}}{\sigma^{2}+\mathcal{I}},
$$

where $P$ is the transmit power level of $\mathbf{p}_{i}, \lambda$ is the RF wavelength, $h_{i j} \sim \mathcal{C N}(0,1)$ is the Rayleigh fading coefficient, $\sigma^{2}$ is the thermal noise power and $\mathcal{I}$ is the interference power level at the receiver as given by

$$
\mathcal{I}=\zeta d_{j}^{-\eta}\left|h_{j}\right|^{2}
$$

where $\zeta=P_{I} G^{2}\left(\frac{\lambda}{4 \pi}\right)^{2}$ and $h_{j} \sim \mathcal{C N}(0,1)$, with $P_{I}$ denoting the transmit power of the interferer. We will then have a connection probability, conditioned on the interference, defined by

$$
\begin{aligned}
& \left.c\right|_{\mathcal{I}}\left(\mathbf{p}_{i}, \mathbf{p}_{j}\right)=\mathbb{P}\left[\operatorname{SINR}_{\mathrm{j}} \geqslant \tau\right] \\
& =\mathbb{P}\left[\frac{P G^{2}\left(\frac{\lambda}{4 \pi}\right)^{2} d_{i j}^{-\eta}\left|h_{i j}\right|^{2}}{\sigma^{2}} \geqslant \tau\left(1+\frac{\mathcal{I}}{\sigma^{2}}\right)\right],
\end{aligned}
$$

where $\tau$ is the minimum SINR required to establish a communication link. For convenience, we define a fixed connectivity scale $r_{0}$ given by

$$
r_{0}^{\eta}=\frac{P G^{2}\left(\frac{\lambda}{4 \pi}\right)^{2}}{\sigma^{2} \tau} .
$$

Since $\left|h_{i j}\right|^{2}$ has a standard exponential distribution, (17) becomes

$$
\left.c\right|_{\mathcal{I}}\left(\mathbf{p}_{i}, \mathbf{p}_{j}\right)=\exp \left(-\frac{d_{i j}^{\eta}}{r_{0}^{\eta}}\left(1+\frac{\mathcal{I}}{\sigma^{2}}\right)\right) .
$$

We now average over the fading variable $\left|h_{j}\right|^{2}$ in the interfering path. Since $\left|h_{j}\right|^{2}$ also has standard exponential distribution, the connection probability is given by

$$
\begin{aligned}
c\left(\mathbf{p}_{i}, \mathbf{p}_{j}\right) & =\int_{0}^{\infty} \exp \left(-\frac{d_{i j}^{\eta}}{r_{0}^{\eta}}\left(1+k d_{j}^{-\eta}\left|h_{j}\right|^{2}\right)\right) \\
& \times \exp \left(-\left|h_{j}\right|^{2}\right) \mathrm{d}\left|h_{j}\right|^{2} \\
& =\mathrm{e}^{-\left(\frac{d_{i j}}{r_{0}}\right)^{\eta}}\left[\frac{1}{1+\Omega}\right]
\end{aligned}
$$

where $\Omega=k d_{i j}^{\eta} d_{j}^{-\eta} / r_{0}^{\eta}$ and $k=\zeta / \sigma^{2}$. Hereafter, with a slight abuse of terminology, we refer to $k$ as the normalised interference-to-noise ratio (INR) ${ }^{6}$.

We proceed with the steps outlined in section III-C to develop the continuum model further. Having defined our

\footnotetext{
${ }^{6}$ In fact, $k$ is proportional to INR, where the proportionality constant is related to effective antenna aperture.
} 
connectivity function, we consider two points close to each other such that

$$
\begin{aligned}
& \log c\left(\mathbf{p}(z), \mathbf{p}\left(z+\delta_{z}\right)\right)=-\left[\frac{1}{r_{0}^{\eta}}\left\|\mathbf{p}\left(z+\delta_{z}\right)-\mathbf{p}(z)\right\|^{\eta}\right. \\
& \left.+\log \left(1+\frac{k}{r_{0}^{\eta}} \frac{\left\|\mathbf{p}\left(z+\delta_{z}\right)-\mathbf{p}(z)\right\|^{\eta}}{\left\|\mathbf{p}\left(z+\delta_{z}\right)\right\|^{\eta}}\right)\right]
\end{aligned}
$$

Using the Cartesian coordinate system and expanding (21) for small $\delta_{z}$ yields

$$
\begin{aligned}
& \log c\left(\mathbf{p}(z), \mathbf{p}\left(z+\delta_{z}\right)\right)= \\
& {\left[1+\frac{k}{\left(x^{2}+y^{2}\right)^{\frac{\eta}{2}}}\right] \frac{\left(\dot{x}^{2}+\dot{y}^{2}\right)^{\frac{\eta}{2}}}{r_{0}^{\eta}} \delta_{z}^{\eta}+O\left(\delta_{z}^{\eta+1}\right) .}
\end{aligned}
$$

For convenience we omit the argument $z$ in functions $x$ and $y$ such that, e.g., $x:=x(z)$ and $\dot{x}:=\dot{x}(z)$. The dot notation denotes the first derivative of the function with respect to its argument, e.g. $\dot{x}:=\frac{\mathrm{d} x(z)}{\mathrm{d} z}$. We define

$$
\mathrm{d} l=\sqrt{\dot{x}^{2}+\dot{y}^{2}} \delta_{z}
$$

to be the path length differential. Then, under the condition that $\mathrm{d} l^{\eta-1} / r_{0}^{\eta} \rightarrow \theta \in \mathbb{R}_{+}$as $\delta_{z} \rightarrow 0$, the limit becomes

$$
q(z)=\theta\left[1+\frac{k}{\left(x^{2}+y^{2}\right)^{\frac{\eta}{2}}}\right] \sqrt{\dot{x}^{2}+\dot{y}^{2}} .
$$

For the special case $\eta=2$, the ratio $\mathrm{d} l / r_{0}^{2}$ is proportional to the connection density, which is defined as the average number of connections per unit of length. Hence, the condition $\mathrm{d} l / r_{0}^{2} \rightarrow \theta$ indicates that the connection density must be constant in the limit.

For convenience we introduce the function

$$
\mu(x, y):=\left[1+\frac{k}{\left(x^{2}+y^{2}\right)^{\frac{\eta}{2}}}\right]^{-1} .
$$

Specifically, the function $\mu: \mathbb{R}^{2} \rightarrow \mathbb{R}_{+}$signifies the link quality at a given point in the plane. We can now write the outage probability as

$$
P_{o}[x, y]=1-\exp \left(-\theta \int_{0}^{1} \frac{\sqrt{\dot{x}^{2}+\dot{y}^{2}}}{\mu(x, y)} \mathrm{d} z\right) .
$$

This formulation provides an expression of the outage probability as a functional ${ }^{7}$ of the parametric path variables $x$ and $y$. We note that the path that minimizes the outage probability is independent of the scaling factor $\theta$.

Remark. It should be noted that performing the same analysis for an unfaded interferer [41], yields the same result. This is intuitive in that one would conjecture that the interference field, over the continuum, would tend to the unfaded mean power level in the limit of infinite relay nodes.

\footnotetext{
${ }^{7}$ The notation $P_{o}[x, y]$ is used to highlight the fact that the outage probability depends on the functions $x$ and $y$.
}

\section{A. Stationary Paths}

Our objective now is to determine the functions $x$ and $y$ that yield a global minimum in the functional (26). For convenience, we cast the outage probability expression in polar coordinates to obtain

$$
P_{o}[r]=1-\exp \left(-\theta \int_{\phi_{1}}^{\phi_{2}} \frac{\sqrt{r^{2}+\dot{r}^{2}}}{\mu(r)} \mathrm{d} \phi\right),
$$

where the link quality function can be expressed as

$$
\mu(r)=\left[1+\frac{k}{r^{\eta}}\right]^{-1} .
$$

The radius $r$ is a function of the angle $\phi$, and $\phi_{1}$ and $\phi_{2}$ represent the respective angle-ordinates of the source and destination nodes.

We now proceed to apply tools from the calculus of variations [42] to compute the stationary paths in polar coordinates, which are defined by the function $r(\phi)$. Denoting the integrand in (26) by $L(r, \dot{r})$ and recognizing that it does not contain any explicit function of $\phi$, we invoke the Beltrami identity to determine the stationary paths:

$$
L-\dot{r} \frac{\partial L}{\partial \dot{r}}=C_{1},
$$

where $C_{1}$ is a constant. Evaluating (29) gives

$$
\dot{r}=\frac{1}{C_{1}} \sqrt{r^{4-2 \eta}\left(k+r^{\eta}\right)^{2}-C_{1}^{2} r^{2}} .
$$

For the special case $\eta=2$ and where $C_{1} \geqslant 2 \sqrt{k}$, there exists a closed form solution to (30) as given by the following proposition.

Proposition 1. The optimum route for the single interferer scenario is given by

$$
r(\phi)=\alpha \operatorname{sn}\left( \pm \frac{\gamma\left(\phi-C_{2}\right)}{C_{1}}, \frac{\alpha}{\gamma}\right),
$$

where $\operatorname{sn}(u, \kappa)$ is the Jacobi elliptic function, $\kappa=\alpha / \gamma$ is the elliptic modulus, $\alpha=0.5 C_{1}-0.5 \sqrt{C_{1}^{2}-4 k}, \gamma=$ $0.5 C_{1}+0.5 \sqrt{C_{1}^{2}-4 k}$ and $C_{1}$ and $C_{2}$ are constants. The Jacobi elliptic function is valid over the range $0<\kappa^{2}<1$.

Proof. See Appendix A.

Remark. Although an analytic solution is constrained by the condition $C_{1} \geqslant 2 \sqrt{k}$ in this scenario, numeric solutions to the Euler Lagrange equations can be found generally, as we demonstrate in subsequent sections.

Given fixed boundary conditions, i.e., the locations of the source and destination nodes, we employ Mathematica to determine the constants $C_{1}$ and $C_{2}$ for a given value of $k$. We illustrate the optimum routes for the cases where the terminal nodes are located at Cartesian coordinates $(1,0),(-1,0)$ and $(0.5,0),(0,1)$, for a range of values of $k$ in Figs. 2 and 3, respectively. The associated values for the constants $C_{1}$ and $C_{2}$ are given in Table I.

It can be argued that the paths shown in Figs. 2 and 3 are all local minima, since one can imagine perturbations of the paths toward the interference source would increase 


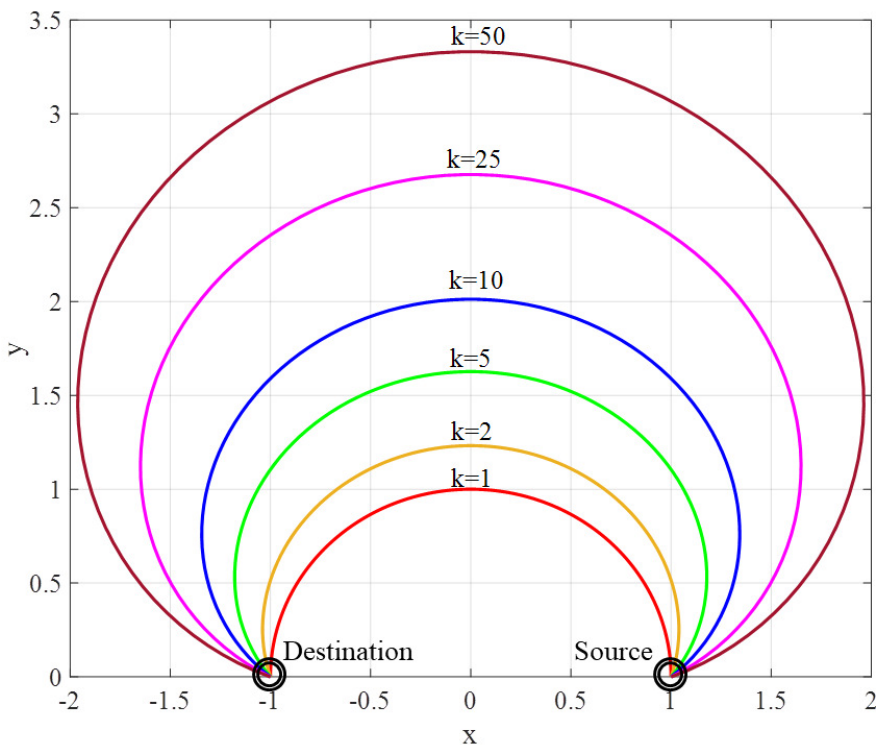

Fig. 2: Optimal routes with single interferer located at $(0,0)$ for $k \in\{1,3,5,10,25,50\}$, Terminal nodes located at Cartesian coordinates $(1,0)$ and $(-1,0)$.

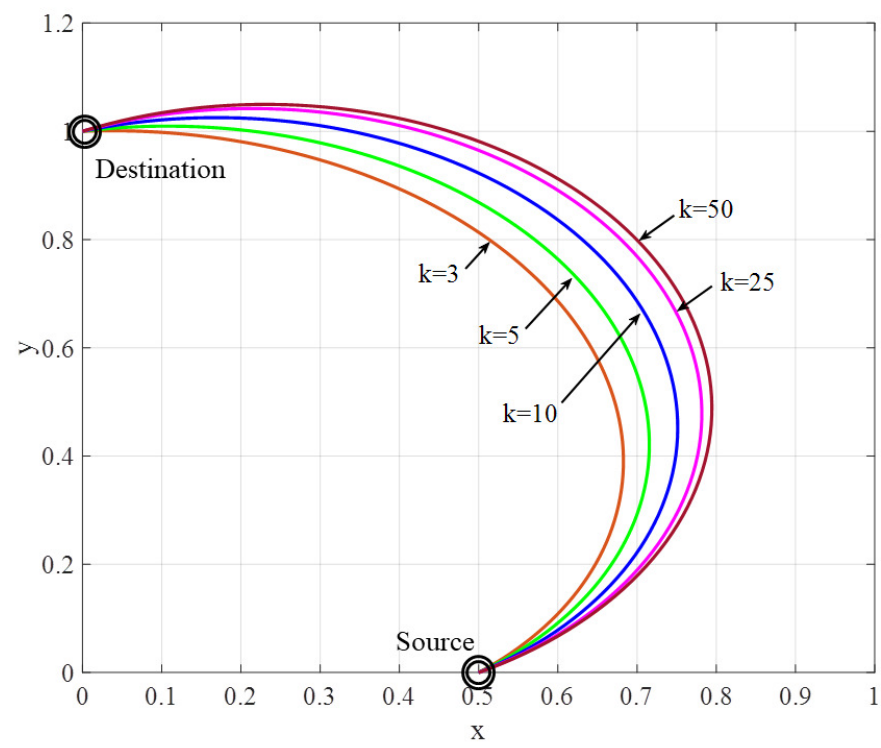

Fig. 3: Optimal routes with single interferer located at $(0,0)$ for $k \in\{3,5,10,25,50\}$ Terminal nodes located at Cartesian coordinates $(0.5,0)$ and $(0,1)$.

TABLE I: Constants $C_{1}$ and $C_{2}$ associated with Fig. 2 (Left most pair) \& Fig. 3 (Right most pair).

\begin{tabular}{ccccc}
\hline \hline$k$ & $C_{1}$ & $C_{2}$ & $C_{1}$ & $C_{2}$ \\
\hline 1 & 2 & -18.4508 & - & - \\
3 & 3.5462 & -1.37672 & 3.99392 & -0.700254 \\
5 & 4.70049 & -1.03243 & 5.90698 & -0.618215 \\
10 & 6.98186 & -0.73590 & 10.5208 & -0.548211 \\
25 & 12.0172 & -0.49360 & 24.0634 & -0.500087 \\
50 & 18.3418 & -0.37286 & 46.4783 & -0.482431 \\
\hline
\end{tabular}

the INR, while perturbations away from the source would lead to a reduction in the connectivity probability (due to the increased path length). More formally, we show that the stationary paths, corresponding to our functional, are minima since they satisfy Legendre's condition. This is detailed in the following proposition.

Proposition 2. Satisfying Legendre's condition [42] provides formal proof that the stationary paths, which satisfy the Euler Lagrange equation, correspond to minima.

Proof. See Appendix B.

\section{B. Single Directional Interferer}

We now introduce a gain function $g:=g(\phi)$ into the functional (27), which permits a non-uniform spatial distribution of interference. The functional can now be written as

$$
P_{o}[r]=1-\exp \left(-\theta \int_{\phi_{1}}^{\phi_{2}}\left[1+\frac{k g}{r^{\eta}}\right] \sqrt{r^{2}+\dot{r}^{2}} \mathrm{~d} \phi\right) .
$$

Since the Beltrami identity is no longer valid, with the inclusion of this explicit function, we invoke the Euler-Lagrange equation

$$
\frac{\partial L}{\partial r}=\frac{\mathrm{d}}{\mathrm{d} \phi}\left(\frac{\partial L}{\partial \dot{r}}\right)
$$

to determine the stationary paths of the functional (32), where $L(\phi, r, \dot{r})$ denotes the integrand. For the special case $\eta=2$, we can show that the Euler-Lagrange equation reduces to

$$
F(\phi, r, \dot{r}):=\ddot{r}=r+\frac{2 \dot{r}^{2}}{r}-\frac{(2 g r+\dot{g} \dot{r})\left(1+\left(\frac{\dot{r}}{r}\right)^{2}\right)}{\frac{r^{2}}{k}+g},
$$

where $\dot{g}=\frac{\mathrm{d} g(\phi)}{\mathrm{d} \phi}$. We now suppose the directivity of the interference transmission is given by gain function

$$
g\left(\phi, \phi_{o}\right)=1+\epsilon \cos \left(\phi-\phi_{o}\right), \quad \epsilon \in[0,1] .
$$

This definition gives a simple cardioid pattern in the azimuth variable $\phi$, where $\phi_{o}$ denotes the orientation angle of the peak antenna gain. The parameter $\epsilon$ defines the magnitude of the peak gain, such that $\epsilon=1$ provides a peak power gain of two and $\epsilon=0$ defines an isotropic radiation pattern.

Since, an analytic solution to (34) does not immediately present itself, we employ numerical methods to solve the differential equation here. Specifically, Euler's explicit method [27] lends itself to this example. We consider $S$ discrete samples over the continuum between the source and destination nodes, uniformly distributed over the interval $\left[\phi_{1}, \phi_{2}\right]$, such that the angular sample spacing is $h=\left(\phi_{2}-\phi_{1}\right) /(S+1)$. Let $i \in\{1,2, \ldots, S+2\}$ denote the index of these samples, where $(\phi(i), r(i))$ represent the polar coordinates of the $i$ th sample, and $i=1$ and $i=S+2$ are the source and destination indices, respectively. By this definition, we have that $\phi(1)=\phi_{1}$ and $r(1)$ is the radial distance of the source from the origin.

One may estimate $\dot{r}(1)$ and invoke Euler's explicit method to return the calculated polar coordinates of the path described by (34). We then successively approximate $\dot{r}(1)$, over the 

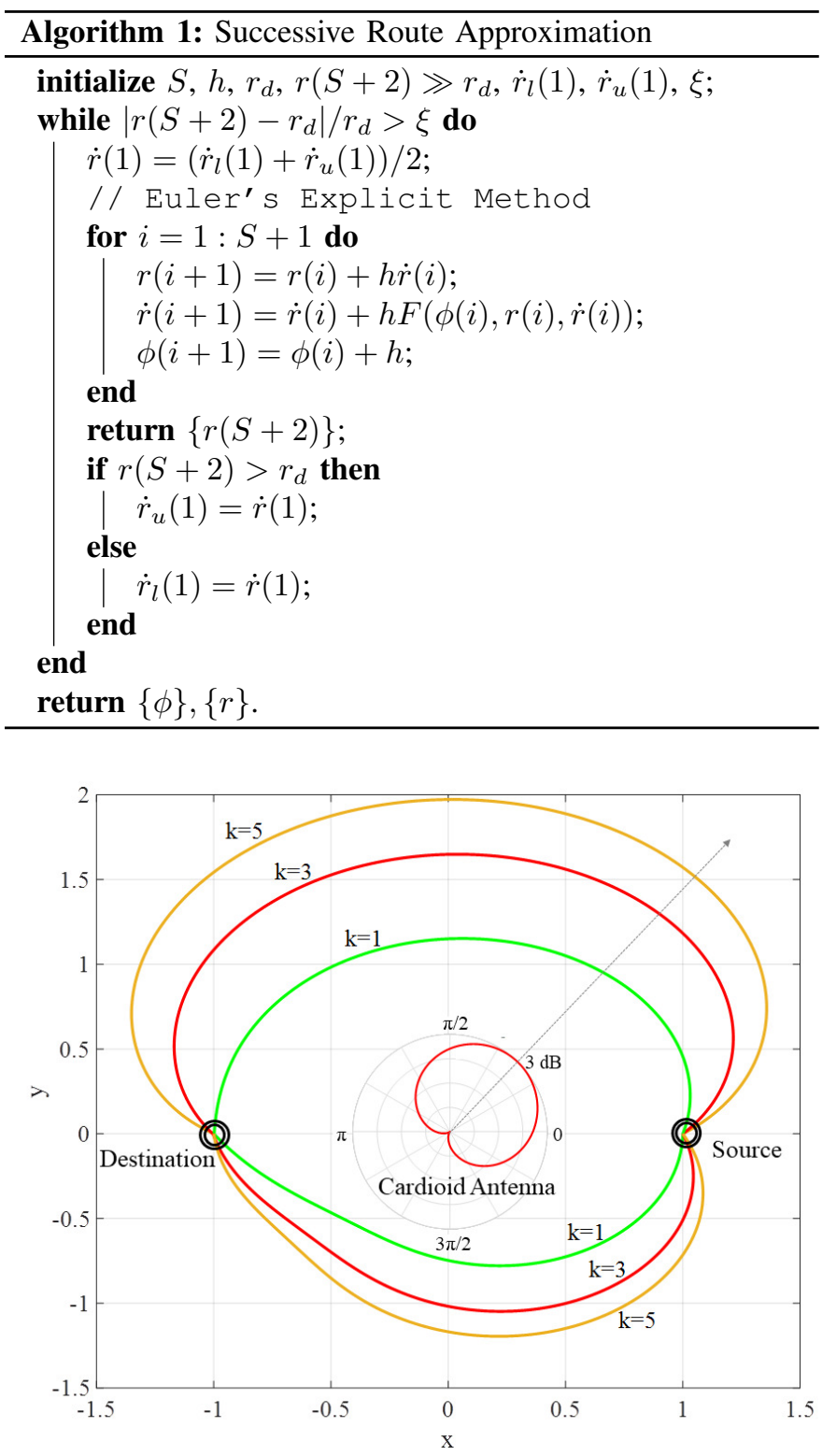

Fig. 4: Optimized routes in the presence of a directional interferer. The directivity is a cardioid pattern with $3 \mathrm{~dB}$ peak gain at $\pi / 4 ; k \in\{1,3,5\}$.

interval $\left[\dot{r}_{l}(1), \dot{r}_{u}(1)\right]^{8}$ until the calculated radial ordinate of the destination node $r(S+2)$ is within an acceptable fractional deviation $\xi$ of the actual radial distance $r_{d}$. Associated pseudocode is outlined in Algorithm 1. It is clear from the description of the algorithm that its complexity does not scale with the number of nodes $N$ in the network; instead, it scales linearly in the number of samples $S$, which may be considerably less than $N$ in large-scale practical scenarios.

As an illustration of this approach, numerically optimized routes for $k \in\{1,3,5\}$ and $\epsilon=1$ are shown in Fig. 4 where

\footnotetext{
${ }^{8}$ The successive approximation is conditional on the variation of $r(S+$ 2 ) being strictly increasing monotonic over the interval $\left[\dot{r}_{l}(1), \dot{r}_{u}(1)\right]$. We determine the interval following a coarse search based upon incrementing $\dot{r}(1)$ over a larger estimated range, invoking Euler's explicit method to return the calculated polar coordinates of the path described by (34) and determine the value of $\dot{r}(1)$ that yields an acceptable approximation of the path.
}

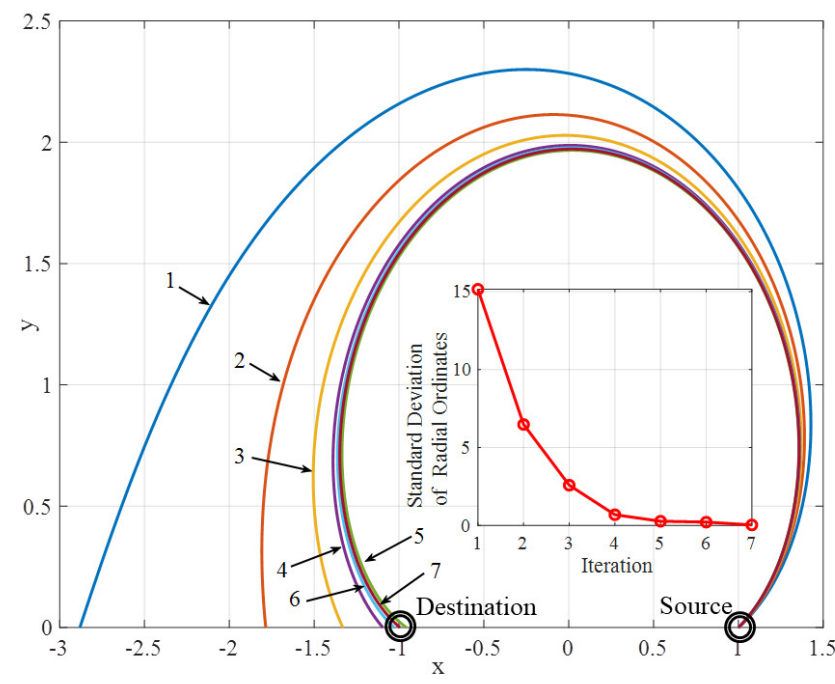

Fig. 5: Illustration of successive route approximations (labelled by iteration number) and standard deviation of radial offset from optimal route for each iteration (inner figure).

$S=1000$. We found that setting $S=50$ gave satisfactory results. With the direction of the peak gain set to $\phi_{o}=\pi / 4$, we clearly observe longer routes between the source and destination nodes in the half-plane interference is directed. We further observe the optimal routes are repelled from the origin in the direction of peak radiation.

Fig. 4 also depicts one path for each value of INR in the lower half-plane. These are also stationary paths that satisfy (34). Interestingly, we see the stationary paths veering toward the interference source in the lower half-plane as a result of the lower level of interference; corresponding to a null in the cardioid antenna pattern. Since the gain function $g$ is independent of $\dot{r}$, the formal test for minima, given in Proposition 2, equally applies to this scenario and provides proof that the stationary paths in the upper and lower half planes of Fig. 4 are minima of the functional (32). These results provide an intuitive illustration of the continuum model in a nontrivial setting with inhomogeneous interference effects.

Fig. 5 provides a visualisation of how the algorithm converges to the final continuum path for the previously described directional interferer scenario, where $k=5, \dot{r}_{l}(1)=1.4$, $\dot{r}_{u}(1)=1.7$ and $\xi=0.01$. The inner figure also shows the rate of convergence of the algorithm, plotting standard deviation of the radial ordinates from the final continuum path as a function of iteration number of consecutive route approximations. The figure shows that, despite the low complexity of the algorithm, the successive approximations converge to the final continuum path in a relatively low number of iterations.

\section{MULTIPLE InTERFERERS}

We now consider application of the continuum model where the multi-hop communications network is subject to interference from multiple independent point sources located in $\mathbb{R}^{2}$. We consider all interferers are independently subject to Rayleigh fading. The locations of the interferers are given by 
the polar coordinates $\mathbf{p}_{m}=\left(r_{m}, \phi_{m}\right)$, where $m \in M$ and $M$ denotes the index set of interferers. We denote the Euclidean distance between a point at $(r, \phi)$ and $\left(r_{m}, \phi_{m}\right)$ by $d_{m}$ where $d_{m}^{2}=r^{2}+r_{m}^{2}-2 r r_{m} \cos \left(\phi-\phi_{m}\right)$.

If we extend the analysis detailed in section IV to multiple independently faded interferers then (20) becomes

$$
c\left(\mathbf{p}_{i}, \mathbf{p}_{j}\right)=\mathrm{e}^{-\left(\frac{d_{i j}}{r_{0}}\right)^{\eta}} \prod_{m \in M}\left[\frac{1}{1+\Omega_{m}}\right],
$$

where $\Omega_{m}=k d_{i j}^{\eta} d_{m}^{-\eta} / r_{0}^{\eta}$. It is then relatively straighforward to show that the functional for outage probability becomes

$$
\begin{aligned}
& P_{o}[r]=1- \\
& \exp \left(-\theta \int_{\phi_{1}}^{\phi_{2}}\left[1+k \sum_{m \in M} \frac{1}{d_{m}^{\eta}}\right] \sqrt{r^{2}+\dot{r}^{2}} \mathrm{~d} \phi\right) .
\end{aligned}
$$

Remark. It can be shown that, repeating the analysis for unfaded interferers also yields (37). The sum of interference power, from Rayleigh faded interferers, will have a hypoexponential distribution. Conjecturing (as for the single interferer case), that in the limit of infinite relay extent, the interference field tends to mean of the distribution, then this will be equivalent to the sum of unfaded interferers which is consistent with the analysis.

Letting $L$ denote the integrand in (37), considering the case $\eta=2$ and evaluating the Euler-Lagrange equation produces

$$
\begin{aligned}
F(\phi, r, \dot{r}) & :=\ddot{r}=r+\frac{2 \dot{r}^{2}}{r} \\
& -\frac{2 k\left(r^{2}+\dot{r}^{2}\right)\left[\Sigma_{2}\left(r^{2}+\dot{r}^{2}\right)-\Sigma_{3} \dot{r}\right]}{\left(1+k \Sigma_{1}\right) r^{2}},
\end{aligned}
$$

where

$$
\begin{aligned}
\Sigma_{1} & =\sum_{m \in M} \frac{1}{d_{m}^{2}} \\
\Sigma_{2} & =\sum_{m \in M} \frac{r-r_{m} \cos \left(\phi-\phi_{m}\right)}{d_{m}^{4}} \\
\Sigma_{3} & =\sum_{m \in M} \frac{r \dot{r}-r_{m}\left[\dot{r} \cos \left(\phi-\phi_{m}\right)-r \sin \left(\phi-\phi_{m}\right)\right]}{d_{m}^{4}}
\end{aligned}
$$

Again, since an analytic solution to (38) does not immediately present itself, we employ Euler's method to solve for $r$, as outlined previously (see section IV-B).

\section{A. Two Interferers}

We start with a simple scenario with two interferers, with $k=3$, located at polar coordinates $(0,0)$ and $(1, \pi / 2)$. Fig. 6 shows three local minimum paths: paths 1 and 3 are similar to previous observations, but with symmetry about the straight line containing the non-directive interferers, and path 2 passing between the two interferers. This third minima is an expected result in that arbitrary paths directly passing through either of the interferers will yield total outage due to the singularities in (37) at $r=d_{m}, m \in M$, and a path existing between the two interferers will intuitively yield a lower outage probability. It should be noted that, although the path 2 is significantly shorter than the paths 1 and 3 , it will not necessarily yield

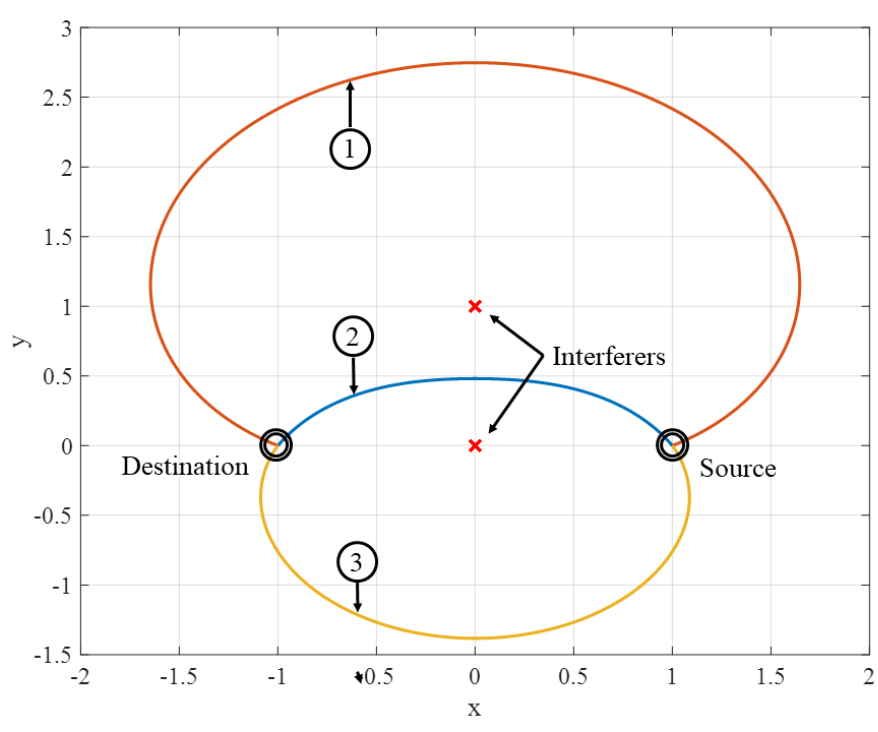

Fig. 6: Optimal routes for 2 interferer scenario. Interferers located at positions $(0,0)$ and $(1, \pi / 2)$, indicated by crosses, and $k=3$. The stationary paths from uppermost to lowermost are labelled 1, 2, and 3 respectively.

the lowest outage probability due to the closer proximity to the interferers. For a given value of $\theta$ we can determine the outage probability by substituting $r$ and $\dot{r}$, corresponding to each stationary path, into (37).

To provide further evidence to support the stationarity of the three paths by an intuitive visualization, we consider a range of arbitrary routes generated by linear interpolation ${ }^{9}$ of radial ordinates of adjacent paths 1,2 , and 3 for fixed angleordinates, and calculate the corresponding outage probability of the interpolated paths. Paths above path 1 and below path 3 are simply generated by scaling paths with the function $\left[1+\Delta r / r_{p}\right] \cos (\phi) . r_{p}$ is the radial ordinate of path- $p \in\{1,3\}$ at respective angle-ordinates $\{\pi / 2,-\pi / 2\}$ and $\Delta r$ is the required deviation of the radial ordinate at respective angleordinates $\{\pi / 2,-\pi / 2\}$. The effect of this route perturbation on outage probability is illustrated in Fig. 7, clearly showing that paths 1,2 , and 3 correspond to minimum stationary paths and total outage occurs when paths pass through an interferer.

\section{B. Three Interferers}

We repeat the previous analysis with three interferers located at polar coordinates $(0,0),(0.5, \pi / 3)$ and $(0.5, \pi / 6)$ with $k \in\{1,2,3\}$. Using Euler's method to solve (38), as before, we illustrate the resultant stationary paths in Fig. 8. The upper and lowermost paths follow similar observations with the single directive interferer scenario; an intuitive result in that the three interferers with spatial bias is analogous to a single interferer located at the origin having increased directivity at angle $\pi / 4$ in this example. We might pursue this analogy to develop a single directive interferer approximation.

\footnotetext{
${ }^{9}$ Linear interpolation provides a straightforward method, and alternative interpolation approaches would be equally applicable.
} 


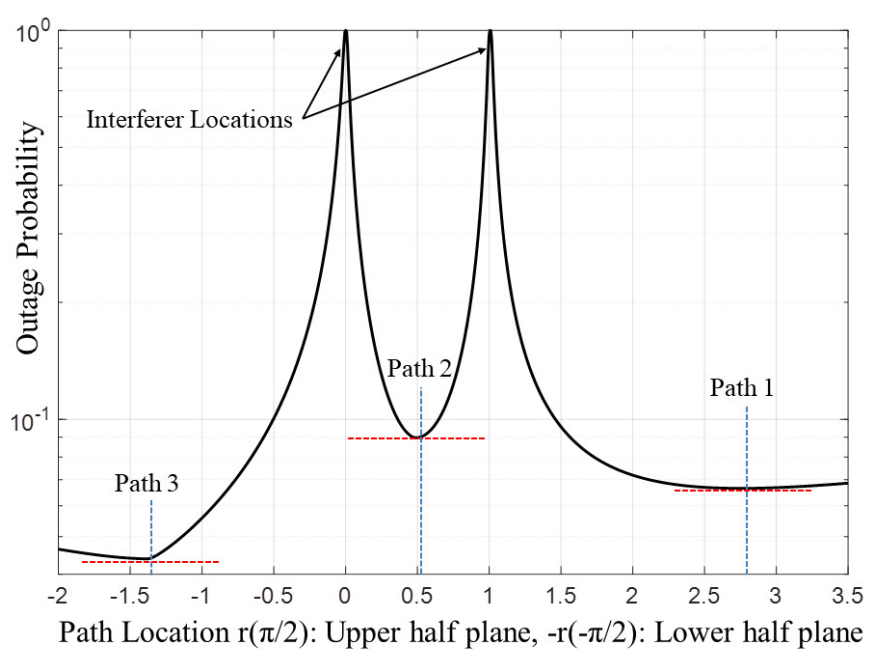

Fig. 7: Verification of path stationarity by route perturbation.

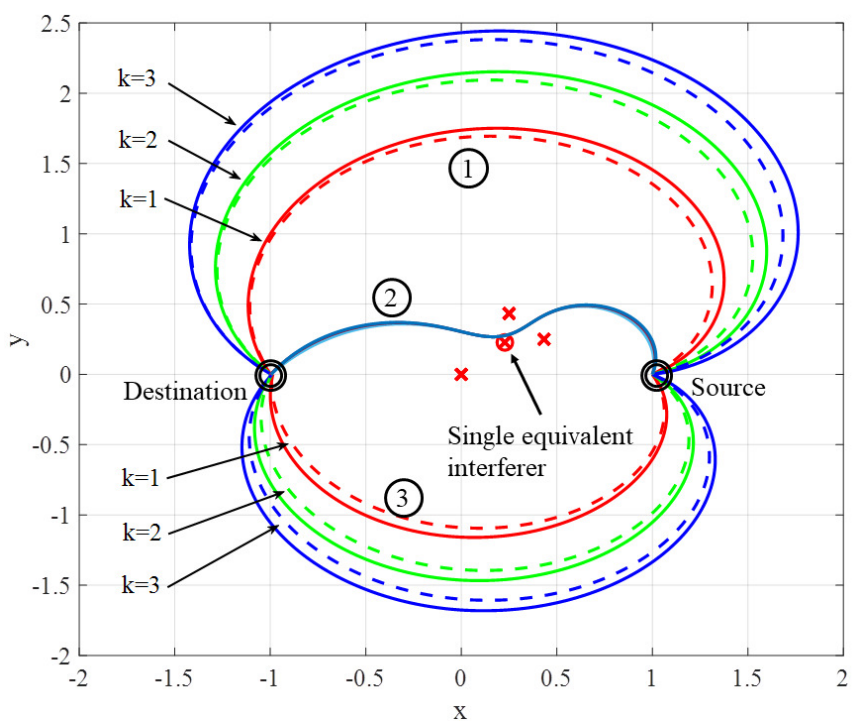

Fig. 8: Stationary paths (solid lines) for 3 interferer scenario for $k \in\{1,2,3\}$. Interferers are located at positions $(0,0)$, $(0.5, \pi / 6),(0.5, \pi / 3)$ (red crosses). The dashed lines represent approximations for the stationary paths for a single equivalent interferer located at position $(0.322, \pi / 4)$.

However, there is little advantage to this approach, since it would not necessarily yield a reduction in processing complexity in determining stationary paths.

Instead, we consider an alternative approximation for a group of interferers. Specifically, one could consider a single non-directive interferer located at an equivalent "centre of interference", given by $\frac{1}{|M|} \sum_{m \in M} \mathbf{p}_{m}$, with an increased INR given by $k^{\prime}=|M| k$. In this example, the single equivalent interferer is located at $(0.322, \pi / 4)$. Using this approximation, we apply (31) to determine stationary paths; avoiding the need for the more cumbersome application of numeric methods in solving (38). The resulting approximate stationary paths are shown as dashed lines in Fig. 8. In this example, the approximations appear to closely match the actual stationary

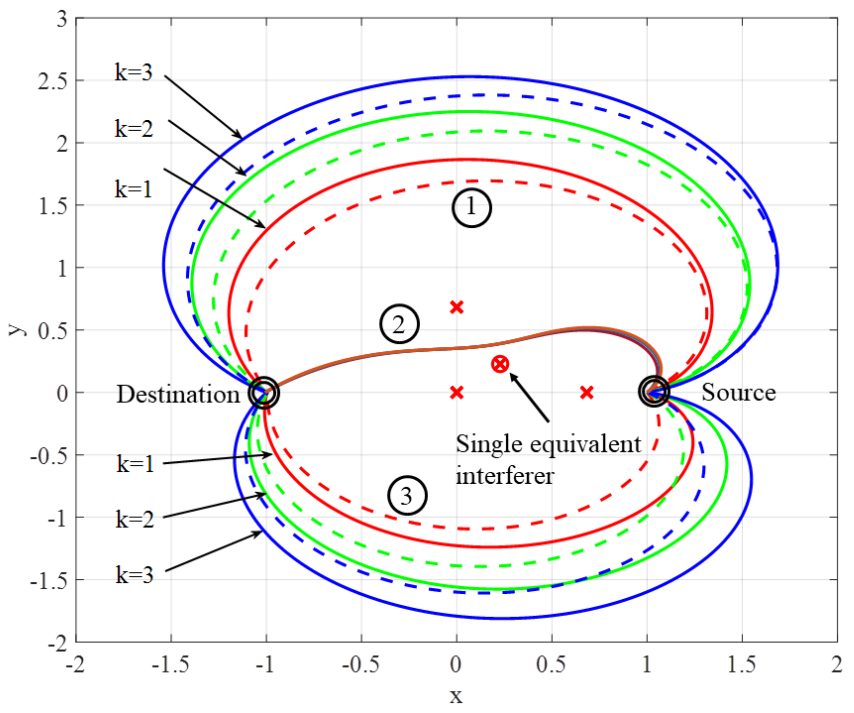

Fig. 9: Stationary paths (solid lines) for 3 interferer scenario for $k \in\{1,2,3\}$. Interferers are located at positions $(0,0)$, $(0.683,0),(0.683, \pi / 2)$ (red crosses). The dashed lines represent approximations for the stationary paths for a single equivalent interferer located at position $(0.322, \pi / 4)$.

paths.

We repeat this analysis for three interferers with increased Euclidean distance between them but with the same equivalent single interferer location as before. The results are shown in Fig. 9, which clearly shows the approximate paths deviate to a larger degree from the actual stationary paths. From this observation, we conjecture that the accuracy of the approximation will degrade with increased variance in Euclidean distances of the multiple interferers from the equivalent "centre of interference". We defer further analysis of this approximation of complex spatial distributions of interferers to future work.

Returning to the three interferers, we observe path 2 meandering between interferers; as observed in the previous two interferer scenario. Further, this path is largely independent of INR close to the interferers, which can be attributed to the effect on the path being analogous to two opposing "forces" equally repelling the path. It is possible to identify other local minima, e.g., meandering from the source towards the lower two interferers and upwards between them and then passing between the left most two interferers towards the destination. However, this path will pass through both lower and upper half planes and $r(\phi)$ becomes non-injective, requiring a change in coordinate system, i.e., parametric Cartesian. Again, we defer treatment of more complex scenarios to future work.

\section{SAMPling the ContinuUm for Practical DEPLOYMENTS}

Having determined a methodology for the creation of an equivalent continuous multi-hop route, we now distribute a finite number of relay nodes onto the stationary path, according to four placement strategies. Here, we list these in increasing order of processing complexity.

- Equi-angle: nodes distributed with constant angular offset 
- Equi-spacing: nodes distributed with constant distance along the continuum

- Equi-Euclidean: nodes distributed with constant Euclidean distance

- Optimum-angle: optimal angle ordinates of nodes are

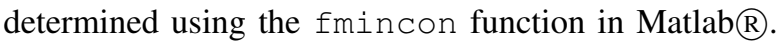

We consider the single non-directive interferer scenario discussed in section IV and assume that an outage probability of 0.05 is a realistic target in a practical deployment. We firstly determine the value of $\theta$ by rearranging (27) such that

$$
\theta=-\frac{\log \left(1-P_{o}[r]\right)}{I},
$$

where $I$ denotes the integral. We then calculate the length scale

$$
r_{o}=\sqrt{\frac{L}{\theta}}
$$

where $L=\int_{\phi_{1}}^{\phi_{2}} \sqrt{r^{2}+\dot{r}^{2}} \mathrm{~d} \phi$ is the length of the continuum. The discrete multi-hop outage probability, employing $N$ relays, is then calculated from

$$
P_{o}=1-\prod_{n=0}^{N} \exp \left(-\frac{N+1}{r_{0}^{2}} \cdot \frac{\|\mathbf{p}(n+1)-\mathbf{p}(n)\|^{2}}{\mu(\|\mathbf{p}(n+1)\|)}\right),
$$

where the link quality function $\mu(\cdot)$ is given by (28) with $\eta=2$. We vary $N$ over the interval $[5,30]$ and calculate the corresponding outage probabilities for each placement strategy for $k=5$. Results are shown in Fig. 10 .

With increasing numbers of nodes, the equi-Euclidean relay spacing converges to the equi-spacing case, which represents uniform relay spacing over the continuum and which satisfies the constant connectivity density condition that underpins our continuum model. In this case, we observe a rapid convergence to the $P_{o}=0.05$ asymptote. Since the equi-angle and optimum angle scenarios violate the constant connection density condition, they do not exactly converge to the $P_{o}=0.05$ asymptote. Notwithstanding this, the simplest form of relay placement yields an acceptably low deviation from the required outage probability. This is further highlighted in Fig. 11, where we vary the effective SNR from the level that yields an outage probability of 0.05 for the continuum.

The nonuniform angular distribution, according to the optimum-angle placement strategy on the continuum, is illustrated in Fig. 12 for the placement of 10 relay nodes (as indicated by circle markers); we observe that the Euclidean distance between adjacent nodes reduces where the receiving node is closer to the interferer. This yields a lower outage probability due to the interference being nonuniform over the continuum, and reducing the Euclidean distance between adjacent relay nodes subject to higher interference will intuitively yield lower outage probability overall. Fig. 12 also shows node placement for 10 and 50 relay nodes where we allow freedom to optimize both radial and angle ordinates using the

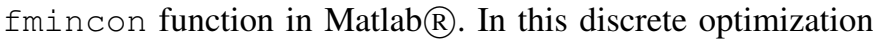
case, we provide an equivalence between the continuum model and the discrete optimization by using the length scale defined by

$$
r_{o}=\sqrt{\frac{\sum_{n=0}^{N}\|\mathbf{p}(n+1)-\mathbf{p}(n)\|}{\theta}},
$$

where $\theta$ is given by (42). This length scale is then substituted into the objective function for outage probability given in (44). In the limit of an infinite number of relays, where we can consider the path of relay nodes forming a continuum, then (45) converges to (43), i.e., as the sum of Euclidean distances for each hop approaches the length of the continuum. We observe that for the placement of 50 nodes, according to this discrete optimization, their locations are close to the continuum, which provides further evidence supporting the utility of the continuum model to optimize relay placement in large multi-hop networks.

In contrast however, the placement of 10 nodes shows a reasonable divergence from the continuum. This observation can be attributed to (45) and its relation to total available transmit power (18), which is equally distributed across the source and relay nodes, i.e., transmit power per relay node is proportional to the sum of Euclidean distances. If the 10 nodes were located on the continuum then the sum of Euclidean distances would be less than the length of the continuum and the nodes would therefore have lower available power, which would result in lower SINR and therefore higher outage probability for the multi-hop network when compared to the continuum. It is intuitive that the optimum placement of relay nodes would be further away from the continuum, which would yield increased transmit power and lower interference, and would mitigate the increase in node separation. This is supported by observations in Fig. 10, where for lower numbers of relays $(N<20)$ the outage probability for the discrete optimization case (depicted by the dashed line) is higher than when relays are located on the continuum according to the optimum-angle placement strategy. As the number of nodes increases, we see a convergence between the two placement strategies.

In this example, the least complex strategy for relay node placement on the continuum does not yield the optimal outage probability for a multi-hop relay network. However, we can conclude that the suboptimality of the continuum approach and relay node placement strategies is not significant.

\section{CONCLUSION AND Discussions}

In this paper, we have demonstrated the effectiveness of the continuum model for end-to-end outage probability calculation when applied to multi-hop route optimization in a wireless network subject to an inhomogeneous scalar field of interference. Taking a canonical example of interference originating from a single point in $\mathbb{R}^{2}$, we have shown that the scale of processing complexity reduces from $O\left(N^{3}\right)$ for a discrete Lagrangian method of constrained optimization (where $N$ is the number of relays) to $O(S)$ in the continuum model, with $S$ denoting the number of samples used to approximate the continuous path. Although analytic forms representing the optimal path do not exist in general, it is possible to employ basic numerical techniques to approximate it. From this approximation, it is straightforward to sample the continuum with a finite number of points (relays), thus yielding an acceptably low deviation in outage probability relative to the optimum.

Several open problems remain. Analytic solutions to particular problems are desirable, and more complicated scenarios 


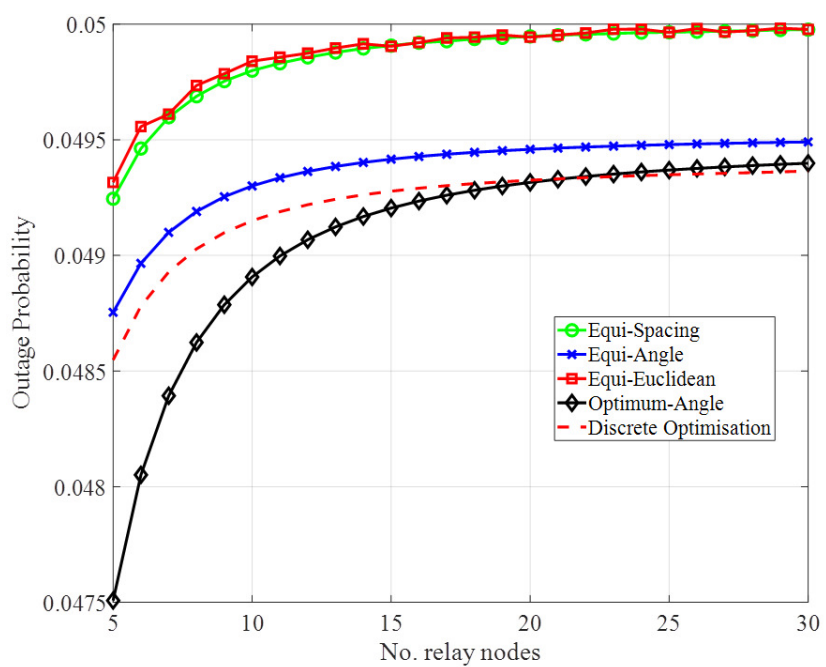

Fig. 10: Outage probability versus the number of relay nodes placed on the continuum according to equi-spacing, equiangle, equi-Euclidean and optimum angle placement strategies for $k=5$. The dashed line represents the outage probability for a relay placement according to the discrete optimization of both radial and angular ordinates of relay nodes where $k=5$.

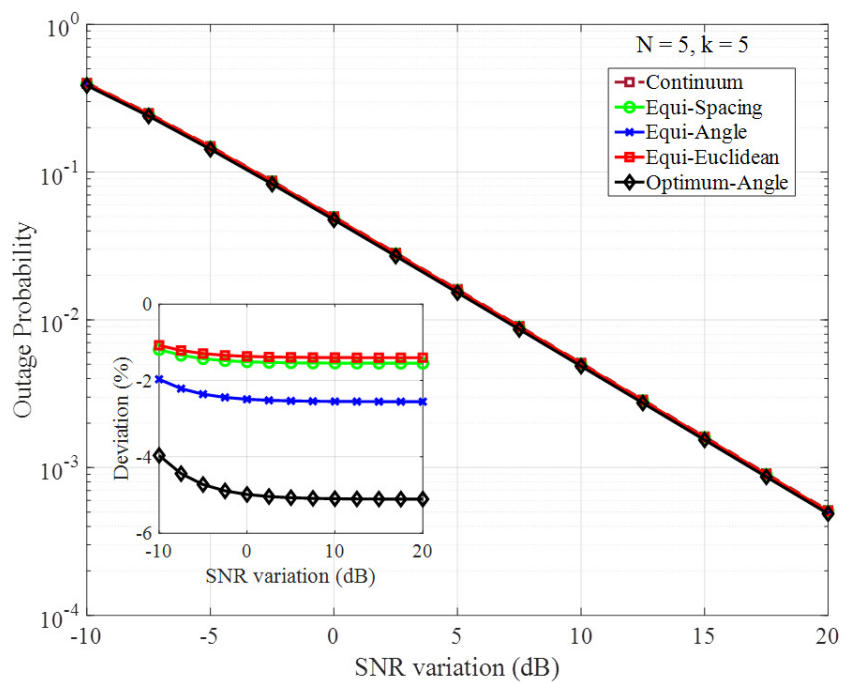

Fig. 11: Outage probability and \% deviation in outage probability from the continuous path versus SNR for five relay nodes placed on the continuous path according to equi-spacing, equiangle, equi-Euclidean, and optimum angle placement strategies for $k=5$.

and cost functions should be considered. Furthermore, optimal sampling of the continuum is also of interest. We hope this work inspires others to consider continuum modelling in related problems in communications and signal processing.

\section{APPENDIX A}

PROOF OF PROPOSITION 1

Substituting $\eta=2$ into (30) and simplifying results in

$$
\dot{r}=\frac{1}{C_{1}} \sqrt{\left(k+r^{2}\right)^{2}-C_{1}^{2} r^{2}} .
$$

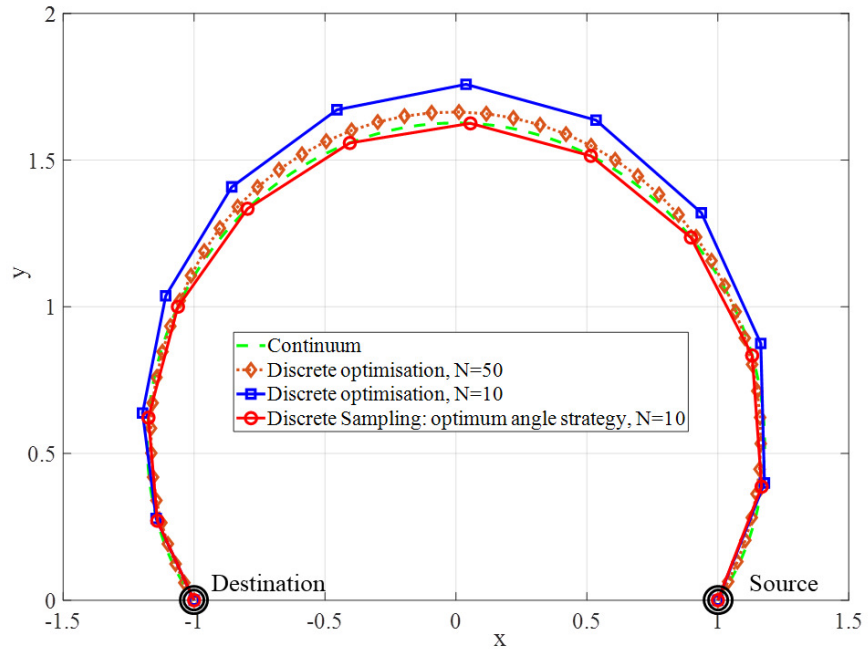

Fig. 12: An example of the placement of 10 relay nodes according to the optimum-angle placement strategy (circle marker representing relay node location) and optimizing both radial and angle ordinates of 10 and 50 relay nodes (relay node locations represented by square and diamond markers respectively).

Separating variables and integrating yields

$$
\phi=C_{1} \int \frac{1}{\sqrt{\left(k+r^{2}\right)^{2}-C_{1} r^{2}}} \mathrm{~d} r .
$$

Defining the denominator in the integrand by function $s(r)$ we have

$$
s(r)=\left(k+r^{2}+C_{1} r\right)\left(k+r^{2}-C_{1} r\right),
$$

and we can define the roots of $s(r)$ by

$$
r= \pm \frac{C_{1}}{2} \pm \frac{1}{2} \sqrt{C_{1}^{2}-4 k} .
$$

If we denote these roots by $\alpha, \beta, \gamma, \delta$ then

$$
\phi=C_{1} \int \frac{1}{\sqrt{r-\alpha} \sqrt{r-\beta} \sqrt{r-\gamma} \sqrt{r-\delta}} \mathrm{d} r .
$$

Since $\alpha=-\beta$ and $\gamma=-\delta$ we can write

$$
\begin{aligned}
\phi & =C_{1} \int \frac{1}{\sqrt{\left(\alpha^{2}-r^{2}\right)\left(\gamma^{2}-r^{2}\right)}} \mathrm{d} r \\
& =C_{1} \frac{1}{\alpha \gamma} \int \frac{1}{\sqrt{\left(1-\left(\frac{r}{\alpha}\right)^{2}\right)\left(1-\left(\frac{r}{\gamma}\right)^{2}\right)}} \mathrm{d} r .
\end{aligned}
$$

Let $t=\frac{r}{\alpha}$; then $\mathrm{d} r=\alpha \mathrm{d} t$, and we have

$$
\phi=C_{1} \frac{1}{\gamma} \int \frac{1}{\sqrt{\left(1-t^{2}\right)\left(1-\kappa^{2} t^{2}\right)}} \mathrm{d} t,
$$

where $\kappa=\frac{\alpha}{\gamma}$ is the elliptic modulus. Given the integral in (52) is the elliptic integral of the first kind, we have

$$
\phi(r)= \pm \frac{C_{1}}{\gamma} \mathrm{F}\left(\sin ^{-1} \frac{r}{\alpha}, \frac{\alpha}{\gamma}\right)+C_{2},
$$

where $u=\mathrm{F}(\psi, \kappa)$ is the elliptic integral of the first kind in Legendre normal form and $C_{2}$ is a constant. Now, given that 
$\psi=\mathrm{F}^{-1}(u, \kappa)=\operatorname{am}(u, \kappa)$ is the Jacobi amplitude, we can write

$$
r(\phi)=\alpha \sin \left[\operatorname{am}\left( \pm \frac{\gamma\left(\phi-C_{2}\right)}{C_{1}}, \frac{\alpha}{\gamma}\right)\right],
$$

which can be written in the form

$$
r(\phi)=\alpha \operatorname{sn}\left( \pm \frac{\gamma\left(\phi-C_{2}\right)}{C_{1}}, \frac{\alpha}{\gamma}\right),
$$

where $\operatorname{sn}(u, \kappa)$ is the Jacobi elliptic function.

\section{APPENDIX B}

\section{PROOF OF PROPOSITION 2}

Given a functional

$$
P_{o}[r]=1-\exp \left(-\theta \int_{\phi_{1}}^{\phi_{2}} \frac{\sqrt{r^{2}+\dot{r}^{2}}}{\mu(r)} \mathrm{d} \phi\right),
$$

we consider the second variation to determine whether the stationary paths, that satisfy the Euler-Lagrange equation, correspond to minima. Specifically, we invoke Legendre's condition ${ }^{10}$,

$$
J(r)=\frac{\partial^{2} L}{\partial \dot{r}^{2}}>0
$$

as sufficient proof of minima; $L$ denoting the integrand of (56). Evaluating $J(r)$, gives

$$
J(r)=\frac{1}{\mu}\left[\frac{1}{\sqrt{r^{2}+\dot{r}^{2}}}-\frac{\dot{r}^{2}}{\left(r^{2}+\dot{r}^{2}\right)^{\frac{3}{2}}}\right] .
$$

Since $\mu(r) \in \mathbb{R}_{+}$, the condition (57) is satisfied when the right hand bracketed expression of (58) is positive, which reduces to the condition $r>0$. Therefore, since $r \in \mathbb{R}_{+}$, the stationary paths correspond to strict minima of the functional.

\section{REFERENCES}

[1] N. Bhushan, J. Li, D. Malladi, R. Gilmore, D. Brenner, A. Damnjanovic, R. Sukhavasi, C. Patel, and S. Geirhofer, "Network densification: the dominant theme for wireless evolution into 5G," IEEE Communications Magazine, vol. 52, no. 2, pp. 82-89, 2014.

[2] J. G. Andrews, X. Zhang, G. D. Durgin, and A. K. Gupta, "Are we approaching the fundamental limits of wireless network densification?" IEEE Communications Magazine, vol. 54, no. 10, pp. 184-190, 2016.

[3] J. Liu, M. Sheng, L. Liu, and J. Li, "Network densification in 5G: From the short-range communications perspective," IEEE Communications Magazine, vol. 55, no. 12, pp. 96-102, 2017.

[4] A. Al-Fuqaha, M. Guizani, M. Mohammadi, M. Aledhari, and M. Ayyash, "Internet of Things: A survey on enabling technologies, protocols, and applications," IEEE Communications Surveys Tutorials, vol. 17, no. 4, pp. 2347-2376, Fourthquarter 2015.

[5] D. Lund, C. MacGillivray, V. Turner, and M. Morales, "Worldwide and regional internet of things (IoT) 2014-2020 forecast: A virtuous circle of proven value and demand,' International Data Corporation (IDC), Tech. Rep, vol. 1, 2014.

[6] J. M. C. Brito, "Trends in wireless communications towards 5G networks; the influence of e-health and IoT applications," in Computer and Energy Science (SpliTech), International Multidisciplinary Conference on. IEEE, 2016, pp. 1-7.

[7] G. Chen, J. P. Coon, A. Mondal, B. Allen, and J. A. Chambers, "Performance analysis for multi-hop full-duplex IoT networks subject to poisson distributed interferers," IEEE Internet of Things Journal, 2018.

[8] W. Zhang, Y. Chen, Y. Yang, X. Wang, Y. Zhang, X. Hong, and G. Mao, "Multi-hop connectivity probability in infrastructure-based vehicular networks," IEEE Journal on Selected Areas in Communications, vol. 30, no. 4, pp. 740-747, May 2012.

${ }^{10}$ Non Euclidean geometries would also need to satisfy the conjugate point condition. However, conjugate points do not exist in Euclidean space [42].
[9] F. Jameel, Z. Hamid, F. Jabeen, S. Zeadally, and M. A. Javed, "A survey of device-to-device communications: Research issues and challenges," IEEE Communications Surveys Tutorials, vol. 20, no. 3, pp. 2133-2168, thirdquarter 2018.

[10] G. Chen, J. P. Coon, and S. E. Tajbakhsh, "Secure routing for multihop ad hoc networks with inhomogeneous eavesdropper clusters," IEEE Transactions on Vehicular Technology, vol. 67, no. 11, pp. 10660 10670 , Nov. 2018.

[11] G. Chen, J. Tang, and J. P. Coon, "Optimal routing for multihop socialbased D2D communications in the internet of things," IEEE Internet of Things Journal, vol. 5, no. 3, pp. 1880-1889, June 2018.

[12] M. Younis and K. Akkaya, "Strategies and techniques for node placement in wireless sensor networks: A survey," Ad Hoc Networks, vol. 6, no. 4, pp. 621 - 655, 2008. [Online]. Available: http://www.sciencedirect.com/science/article/pii/S1570870507000984

[13] M. Minelli, M. Ma, M. Coupechoux, J. Kelif, M. Sigelle, and P. Godlewski, "Optimal relay placement in cellular networks," IEEE Transactions on Wireless Communications, vol. 13, no. 2, pp. 998-1009, February 2014.

[14] M. Zolotukin, A. Sayenko, and T. Hamalainen, "On optimal relay placement for improved performance in non-coverage limited scenarios," in Proceedings of the 17th ACM International Conference on Modeling, Analysis and Simulation of Wireless and Mobile Systems, ser. MSWiM '14. New York, NY, USA: ACM, 2014, pp. 127-135. [Online]. Available: http://doi.acm.org/10.1145/2641798.2641817

[15] S. Y. Lee and T. Kim, "Optimal relay station placement with non-uniform user distribution," Wireless Personal Communications, vol. 107, no. 1, pp. 121-136, Jul 2019. [Online]. Available: https://doi.org/10.1007/s11277-019-06243-y

[16] M. Minelli, M. Maode, M. Coupechoux, J. Kelif, M. Sigelle, and P. Godlewski, "Uplink energy-delay trade-off under optimized relay placement in cellular networks," IEEE Transactions on Mobile Computing, vol. 15, no. 9, pp. 2376-2387, Sep. 2016.

[17] A. Efrat, S. Har-Peled, and J. S. Mitchell, "Approximation algorithms for two optimal location problems in sensor networks," in 2nd International Conference on Broadband Networks, 2005. IEEE, 2005, pp. 714-723.

[18] X. Cheng, D.-Z. Du, L. Wang, and B. Xu, "Relay sensor placement in wireless sensor networks," Wireless Networks, vol. 14, no. 3, pp. 347355, 2008.

[19] S. Poduri, S. Pattem, B. Krishnamachari, and G. S. Sukhatme, "Sensor network configuration and the curse of dimensionality," in Proc. Third Workshop on Embedded Networked Sensors (EmNets 2006), Cambridge, MA, USA. Citeseer, 2006.

[20] A. Mazumder, Chenyang Zhou, A. Das, and A. Sen, "Budget constrained relay node placement problem for maximal "connectedness"," in $M I L$ COM 2016 - 2016 IEEE Military Communications Conference, Nov 2016, pp. 849-854.

[21] X. Gao, J. Lu, H. Wang, F. Wu, and G. Chen, "Algorithm design and analysis for wireless relay network deployment problem," IEEE Transactions on Mobile Computing, 2018.

[22] G. Kumar and V. Ranga, "Meta-heuristics for relay node placement problem in wireless sensor networks," in 2016 Fourth International Conference on Parallel, Distributed and Grid Computing (PDGC), Dec 2016, pp. 375-380.

[23] J. M. Lanza-Gutiérrez, N. Caballé, J. A. Gómez-Pulido, B. Crawford and R. Soto, "Toward a robust multi-objective metaheuristic for solving the relay node placement problem in wireless sensor networks," Sensors, vol. 19, no. 3, p. 677, 2019.

[24] S. A. Cook, "An overview of computational complexity," Commun. $A C M$, vol. 26, no. 6, pp. 400-408, Jun. 1983. [Online]. Available: http://doi.acm.org/10.1145/358141.358144

[25] F. Kuipers, P. Van Mieghem, T. Korkmaz, and M. Krunz, "An overview of constraint-based path selection algorithms for QoS routing," IEEE Communications Magazine, vol. 40, no. 12, pp. 50-55, Dec 2002.

[26] K. M. Alzoubi, Peng-Jun Wan, and O. Frieder, "New distributed algorithm for connected dominating set in wireless ad hoc networks," in Proceedings of the 35th Annual Hawaii International Conference on System Sciences, Jan 2002, pp. 3849-3855.

[27] K. E. Atkinson, An introduction to numerical analysis. John Wiley \& Sons, 2008.

[28] S. Arreckx, A. Lambe, J. R. R. A. Martins, and D. Orban, "A matrix-free augmented lagrangian algorithm with application to largescale structural design optimization," Optimization and Engineering, vol. 17, no. 2, pp. 359-384, Jun 2016. [Online]. Available: https://doi.org/10.1007/s11081-015-9287-9

[29] "Matlab® Documentation Optimization Toolbox, fmincon," http://mathworks.com. 
[30] S. K. Sharma and X. Wang, "Towards massive machine type communications in ultra-dense cellular IoT networks: Current issues and machine learning-assisted solutions,' IEEE Communications Surveys \& Tutorials, 2019.

[31] A. A. Shabana, Computational continuum mechanics. John Wiley \& Sons, 2018.

[32] J. Kelif, M. Coupechoux, and P. Godlewski, "Fluid model of the outage probability in sectored wireless networks," in 2008 IEEE Wireless Communications and Networking Conference, March 2008, pp. 2933 2938.

[33] J.-M. Kelif, M. Coupechoux, and P. Godlewski, "A fluid model for performance analysis in cellular networks," EURASIP J. Wirel. Commun. Netw., vol. 2010, pp. 1:1-1:11, Jan. 2010. [Online]. Available: http://dx.doi.org/10.1155/2010/435189

[34] H. Feng, L. Luo, Y. Wang, M. Ye, and R. Dong, "A novel minimal exposure path problem in wireless sensor networks and its solution algorithm," International Journal of Distributed Sensor Networks, vol. 12, no. 8, p. 1550147716664245, 2016.

[35] H. Elayan, R. M. Shubair, J. M. Jornet, and P. Johari, "Terahertz channel model and link budget analysis for intrabody nanoscale communication," IEEE Transactions on NanoBioscience, vol. 16, no. 6, pp. 491-503, Sep. 2017.

[36] L. Felicetti, M. Femminella, G. Reali, and P. Liò, "Applications of molecular communications to medicine: A survey," Nano Communication Networks, vol. 7, pp. 27 - 45, 2016. [Online]. Available: http://www.sciencedirect.com/science/article/pii/S1878778915000411

[37] M. Jain, J. I. Choi, T. Kim, D. Bharadia, S. Seth, K. Srinivasan, P. Levis, S. Katti, and P. Sinha, "Practical, real-time, full duplex wireless," in Proceedings of the 17th annual international conference on Mobile computing and networking. ACM, 2011, pp. 301-312.

[38] B. Debaillie, D.-J. van den Broek, C. Lavin, B. van Liempd, E. A. Klumperink, C. Palacios, J. Craninckx, B. Nauta, and A. Pärssinen, "Analog/rf solutions enabling compact full-duplex radios," IEEE Journal on Selected Areas in Communications, vol. 32, no. 9, pp. 1662-1673, 2014.

[39] D. Coppersmith and S. Winograd, "Matrix multiplication via arithmetic progressions," Journal of Symbolic Computation, vol. 9, no. 3, pp. 251 - 280, 1990, computational algebraic complexity editorial. [Online]. Available: http://www.sciencedirect.com/science/article/pii/S0747717108800132

[40] C. Cai, Y. Cai, X. Zhou, W. Yang, and W. Yang, "When does relay transmission give a more secure connection in wireless ad hoc networks?" IEEE Transactions on Information Forensics and Security, vol. 9, no. 4, pp. 624-632, April 2014.

[41] D. Hedges, J. Coon, and G. Chen, "A continuum model for route optimisation in large-scale inhomogeneous multi-hop wireless networks," EUSIPCO 2019, 27th European Signal Processing Conference, 2019.

[42] M. Morse, The calculus of variations in the large. American Mathematical Soc., 1934, vol. 18. 